\title{
Métodos eletrocardiográficos em equinos American Miniature Horse
}

\author{
Electrocardiographic methods in equines American Miniature Horse
}

\author{
Gabriela Nascimento Dantas ${ }^{I}$ Maria Lucia Gomes Lourenço ${ }^{I^{*}}$ \\ Bianca Paola Santarosa ${ }^{I}$ Carla Maria Vela Ulian ${ }^{\mathrm{I}}$ Marta Cristina Thomas Heckler \\ Lídia Raquel de Carvalho ${ }^{I I}$ Simone Biagio Chiacchio ${ }^{I}$
}

\section{RESUMO}

O objetivo deste estudo foi analisar e comparar os registros eletrocardiográficos de 50 equinos American Miniature Horse, clinicamente saudáveis, obtidos pelos métodos convencional e computadorizado, para a padronização do método informatizado. As medidas de duração e amplitude das ondas e intervalos e frequência cardiaca foram analisadas nas derivações do plano frontal e na derivação base-ápice. Diferenças quanto à duração da onda $P$, foram encontradas nas derivações I e aVL, e do complexo QRS, em todas as derivações do plano frontal. Quanto à derivação base-ápice, houve diferença na duração do intervalo QT e da onda $T$, assim como na amplitude da onda S. Concluiu-se, portanto, que ocorre superestimação da duração do complexo QRS no método computadorizado e que, devido às diferenças inerentes de cada método, torna-se importante a utilização de valores de referência para o método computadorizado também na espécie equina.

Palavras-chave: cavalo, eletrocardiograma computadorizado, eletrocardiograma convencional, pôneis.

\section{ABSTRACT}

The aim of this study was to analyze and compare electrocardiographic records of 50 horses American Miniature Horse, clinically healthy, obtained by conventional methods and computerized, to the standardization of the computerized method. The measures of duration and amplitude of waves and intervals and heart rate were analyzed in the leads of the frontal plane and base-apical lead. Differences about the duration of the $P$ wave were found in I and aVL derivations, and QRS complex, in all derivations of the frontal plane. As for basis-apical derivation, there was no difference in the duration of the QT interval and the $T$ wave and the amplitude of the $S$ wave, therefore concluding that overestimation occurs in the duration of the QRS complex in computerized method and that due to inherent differences of each method, it becomes important the use of reference values for the computerized method also in the equine species.
Key words: horse, computerized electrocardiogram, conventional electrocardiogram, ponies.

\section{INTRODUÇÃO}

Ouso da eletrocardiografiaéimprescindível para uma avaliação cardíaca completa e os parâmetros eletrocardiográficos apresentam variações entre as diversas raças de equinos (HILWIG, 1977). Porém, estudos eletrocardiográficos em equinos clinicamente saudáveis de diferentes raças ainda são escassos. A orientação, a amplitude e a duração das ondas do eletrocardiograma (ECG) dependem de vários fatores além da raça do animal, como idade, sexo e derivação estudada (REEF, 1992). Além disso, o ECG é um exame complementar de baixo custo, não invasivo e de fácil realização a campo (FREGIN, 1982).

Com o avanço da informática, a eletrocardiografia computadorizada tem sido utilizada em Medicina Veterinária como método de diagnóstico auxiliar, apresentando alto grau de confiabilidade, quando comparada com o método convencional (SHIWEN et al., 1996; WOLF et al., 2000). Desde a década de 1960, quando os primeiros estudos com a eletrocardiografia computadorizada foram publicados, a técnica vem sendo utilizada e difundida amplamente, contudo, os programas de análise computadorizada ainda apresentam limitações (HONGO \& GOLDSCHLAGER, 2006).

\footnotetext{
'Departamento de Clínica Veterinária, Faculdade de Medicina Veterinária e Zootecnia (FMVZ), Universidade Estadual Paulista (UNESP), 18618-970, Botucatu, SP, Brasil. E-mail: mege@fmvz.unesp.br. *Autor para correspondência.

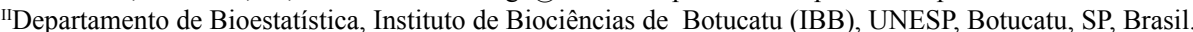


Estudos comparativos entre a eletrocardiografia convencional e computadorizada são escassos na Medicina Veterinária, não havendo na literatura consultada valores de referência em todas as espécies para a eletrocardiografia computadorizada. Segundo WOLF et al. (2000), existem diferenças quanto à amplitude e duração de algumas deflexões do eletrocardiograma entre os métodos em cães, gatos (CAMACHO et al., 2010). Contudo, os padrões de normalidade da eletrocardiografia computadorizada em equinos de diversas raças ainda não foram estabelecidos, particularmente as consideradas de porte pequeno (pôneis), sendo importante considerar que essas diferenças provavelmente existam, assim como em outras espécies, durante a interpretação dos exames.

Os equinos da raça American Miniature Horse possuem a conformação de um cavalo em miniatura, musculoso e proporcional (Associação Brasileira dos Criadores de Mini Horse, 2014), sendo uma raça de tripla aptidão, utilizada tanto para sela como para tração ou simplesmente como animal de estimação. Suas principais peculiaridades são: necessidade de pequenos espaços para mantê-los, baixo custo de criação, porte diminuto, resistência, docilidade e rusticidade, características que fazem com que a representatividade da raça apresente crescimento importante no Brasil.

O objetivo deste estudo foi analisar e comparar o registro eletrocardiográfico de 50 equinos da raça American Miniature Horse, clinicamente saudáveis, obtido pelos métodos convencional e computadorizado, para a padronização do método informatizado na referida raça.

\section{MATERIAL E MÉTODOS}

Foram avaliados 50 equinos da raça American Miniature Horse, 39 fêmeas (78\%) e 11 machos $(22 \%)$, com idades entre quatro meses e 21 anos e peso corporal variando entre $40-190 \mathrm{~kg}$ (média e desvio-padrão 122,9 \pm 31 ). Antes do início do estudo, todos os animais foram submetidos a exame clínico criterioso, constituído por exame físico geral (avaliação de mucosas aparentes, grau de hidratação, tempo de preenchimento capilar, palpação de linfonodos e de pulso arterial e digital, mensuração das frequências cardíaca e respiratória, avaliação da motilidade intestinal, das descargas da válvula íleocecal e aferição de temperatura retal) e avaliação do histórico de cada animal (questionamentos referentes a sinais clínicos presentes atualmente e doenças pregressas).

Para a realização do eletrocardiograma, foram empregados aparelho computadorizado (ECG-
PC versão 2.07, TEB, Brasil) e convencional com papel termossensível milimetrado, próprio para traçados eletrocardiográficos (ECG 6, Ecafix, Brasil). $\mathrm{O}$ registro foi realizado com os animais contidos em local dotado de piso revestido com placas de borracha, com o objetivo de evitar interferências no traçado eletrocardiográfico. Ambos os registros, computadorizado e convencional, foram realizados sequencialmente em cada equino a partir das derivações no plano frontal (I, II, III, aVR, aVL e $\mathrm{aVF}$ ), sendo os eletrodos torácicos direito e esquerdo posicionados acima do olécrano, e os eletrodos pélvicos direito e esquerdo acima dos ligamentos patelares no aspecto cranial de cada membro.

A distribuição dos eletrodos, utilizada para o sistema de derivação bipolar base-ápice, foi a descrita por LOON \& PATTESON (2010), posicionando-se o eletrodo positivo no lado esquerdo, acima do ápice cardíaco, logo atrás do olécrano, e o eletrodo negativo no lado direito, cranial à escápula, próximo à veia jugular e, finalmente, o eletrodo terra, fixado na cernelha do animal.

Os exames foram realizados na velocidade de $50 \mathrm{~mm} /$ segundo e sensibilidade ajustada para $1 \mathrm{~cm}=1$ milivolt $(\mathrm{mV})$, ao longo de um minuto, analisando-se pelo menos 10 complexos em cada eletrocardiograma. Para cada registro eletrocardiográfico, foram analisados a frequência cardíaca, o ritmo, a duração e amplitude da onda P, a duração do intervalo PR, a duração do complexo QRS, a amplitude da onda R, a amplitude da onda S, a duração do intervalo QT, duração e amplitude da onda $\mathrm{T}$ em todas as sete derivações. $\mathrm{O}$ eixo elétrico cardíaco médio expresso em graus foi obtido a partir das derivações I e III, segundo TILLEY (1992).

Para a análise estatística, foi feita comparação somente para o tipo de eletrocardiógrafo, utilizando-se o teste t de Student para populações dependentes. Aanálise de correlação entre a frequência cardíaca e os intervalos PR, QT e complexo QRS, foi feita utilizando-se o teste de Pearson e de Spearman, conforme a distribuição dos dados, em paramétricos e não paramétricos, respectivamente. $\mathrm{O}$ nível de significância utilizado foi de 5\%.

\section{RESULTADOS E DISCUSSÃO}

Todos os animais deste estudo exibiram, em ambos os métodos eletrocardiográficos, os seguintes ritmos cardíacos: $71,11 \%$ (32/45 animais) taquicardia sinusal, $11,11 \%$ (5/45 animais) ritmo sinusal normal, $11,11 \%$ (5/45 animais) arritmia sinusal e 6,6\% (3/45 animais) bradicardia sinusal. Em 
cinco animais, houve discordância entre os métodos em relação à determinação do ritmo cardíaco. Em três equinos, no método computadorizado, primeiramente realizado, a taquicardia sinusal predominou com frequências cardíacas em torno de 62, 86 e $62 \mathrm{bpm}$. Sequencialmente, no método convencional, houve um decréscimo da frequência para 42, 54 e 40, respectivamente, e alteração do ritmo para arritmia sinusal. Em outros dois animais, os ritmos computadorizados foram bradicardia e arritmia sinusal, com frequências de 25 e $40 \mathrm{bpm}$, modificandose ambos para taquicardia sinusal, com elevação das frequências para 63 e $55 \mathrm{bpm}$, no método convencional. Embora tenha ocorrido diferença entre o ritmo cardíaco e a frequência cardíaca de cinco animais nos dois métodos deste estudo, não houve diferença significativa para frequência cardíaca determinada na derivação II do plano frontal e na derivação baseápice. O ritmo cardíaco predominante foi o sinusal, particularmente a taquicardia $(>40 \mathrm{bpm})$. A proporção de arritmias vagal-mediadas não foi predominante $(11,11 \%)$, contrariamente ao descrito pela literatura, como sendo a mais proeminente na espécie equina. Com a contenção dos animais para a realização do ECG e a excitação, houve o predomínio do tônus simpático, prevenindo a ocorrência de tais arritmias, conforme proposto por REZAKHANI et al. (2010).

$\mathrm{Na}$ espécie equina, os pôneis, identificados por um grupo de equinos de baixa estatura de diferentes raças, segundo a Associação Brasileira dos Criadores de Mini-Horse (2012), possuem frequência cardíaca mais elevada que as raças de grande porte (BUSS et al., 1975; SCHWARZWALD et al., 2012). Nos mamíferos, isso se deve, em grande parte, pelo incremento da taxa metabólica, inversamente proporcional ao incremento do peso, sendo o metabolismo mais elevado nos animais de pequeno porte quando comparados aos de grande porte (SCHWARZWALD et al., 2012).

A taquicardia sinusal é provocada por aumento da atividade simpática e, quando é encontrada ao repouso em animais hígidos, ocorre frequentemente em decorrência de excitação e aumento do tônus simpático (LESSA et al., 2012). A caracterização do ritmo como taquicardia sinusal é feita não somente pela presença e regularidade das ondas eletrocardiográficas ( $\mathrm{P}, \mathrm{QRS}, \mathrm{T})$, como também pela aferição da frequência cardíaca. Em equinos adultos saudáveis, os valores da frequência variam de acordo com a literatura. REEF (1992) a descreveu, em equinos de grande porte, como 28 a 44 batimentos por minuto ao repouso e em torno de 70 a $80 \mathrm{bpm}$ em potros.

Raças de pequeno porte, de acordo com SCHWARZWALD et al. (2012), possuem frequência cardíaca e intervalos eletrocardiográficos mais curtos quando comparados às raças de grande porte. Embora a diferença entre os portes possa ser pequena, o peso corporal deve ser considerado ao se avaliar a frequência cardíaca e os intervalos eletrocardiográficos. No entanto, apesar da importância clínica da frequência cardíaca em equinos de diferentes raças e portes, há pouca informação específica sobre os ECGs de pôneis e o limite utilizado para se caracterizar o ritmo como taquicardia sinusal, com base na frequência.

A variação do ritmo cardíaco encontrado neste estudo, de acordo com o método utilizado, ocorreu contrariamente ao descrito na literatura, uma vez que alguns autores identificaram a presença do mesmo ritmo independente do método utilizado. WOLF et al. (2000) descreveram variação de ritmo em cães com presença de arritmia sinusal respiratória, contrariamente ao descrito por CAMACHO et al. (2010) em gatos, observando-se $100 \%$ de similaridade dos ritmos cardíacos entre os métodos. Segundo estes autores e TILLEY (1992), este fato deve-se à alta atividade do componente autônomo simpático em gatos, quando comparado aos cães com predomínio do tônus parassimpático. Em equinos saudáveis, a incidência de arritmias cardíacas ocorre em torno de $30 \%$, sendo, em grande parte, fisiológicas. As arritmias atribuídas ao tônus vagal foram as mais encontradas em equinos saudáveis (ROBERTSON, 1992).

Quanto à duração das ondas e intervalos, verificou-se diferença significativa $(\mathrm{P}<0,0001)$, havendo predomínio de maior duração no ECG computadorizado, da onda $\mathrm{P}$ nas derivações DI e aVL, do complexo QRS, em todas as derivações do plano frontal (bipolares e unipolares aumentadas) e da onda T em I e base-ápice. Os intervalos PR e QT não exibiram diferenças significativas entre os métodos nas derivações do plano frontal, contudo, na derivação base-ápice, a duração da onda T e do intervalo QT apresentaram maior valor no ECG convencional (Tabela 1). Houve, nos dois métodos, predomínio de segmento ST isoelétrico (normodesnivelamento) nas sete derivações analisadas em ambos os métodos.

Os parâmetros como amplitude da onda $\mathrm{P}, \mathrm{R}$ e $\mathrm{T}$ nas derivações do plano frontal (I, II, III, $\mathrm{aVR}, \mathrm{aVL}, \mathrm{aVF}$ ) e na derivação base-ápice não apresentaram diferenças entre o ECG convencional e o computadorizado. A amplitude da onda $\mathrm{S}$ foi avaliada na derivação base-ápice, sendo os valores encontrados, superiores no método computadorizado $(\mathrm{P}<0,0001)$, quando comparado ao convencional (Tabela 2).

Não houve diferença entre o ECG convencional e o computadorizado da frequência cardíaca avaliada nas derivações II do plano frontal e base-ápice, em equinos da raça American Miniature 
Tabela 1 - Duração das ondas, complexos e intervalos eletrocardiográficos de equinos da raça American Miniature Horse, submetidos ao eletrocardiograma convencional e computadorizado, segundo as derivações do plano frontal e base-ápice (média \pm desviopadrão).

\begin{tabular}{|c|c|c|c|c|c|c|c|}
\hline \multicolumn{2}{|c|}{-- Ondas $\left(\mathrm{mm} \mathrm{s}^{-1}\right) \mathrm{I}$----- } & \multirow{2}{*}{$\frac{\text { II }}{0,06 \pm 0,01}$} & \multirow{2}{*}{$\frac{\text { III }}{0,05 \pm 0,01}$} & \multirow{2}{*}{$\frac{\mathrm{aVR}}{0,05 \pm 0,01}$} & \multirow{2}{*}{$\frac{\mathrm{aVL}}{0,04 \pm 0,02^{*}}$} & \multirow{2}{*}{$\frac{\mathrm{aVF}}{0,06 \pm 0,01}$} & \multirow{2}{*}{$\frac{\mathrm{BA}}{0,07 \pm 0,02}$} \\
\hline P Conv & $0,05 \pm 0,01^{*}$ & & & & & & \\
\hline Comp & $0,06 \pm 0,01$ & $0,06 \pm 0,01$ & $0,06 \pm 0,01$ & $0,06 \pm 0,01$ & $0,06 \pm 0,01$ & $0,07 \pm 0,01$ & $0,08 \pm 0,01$ \\
\hline Conv & $0,15 \pm 0,03$ & $0,17 \pm 0,04$ & $0,16 \pm 0,03$ & $0,16 \pm 0,03$ & $0,15 \pm 0,05$ & $0,17 \pm 0,03$ & $0,18 \pm 0,02$ \\
\hline Comp & $0,16 \pm 0,03$ & $0,16 \pm 0,03$ & $0,16 \pm 0,03$ & $0,16 \pm 0,03$ & $0,16 \pm 0,03$ & $0,16 \pm 0,03$ & $0,18 \pm 0,03$ \\
\hline Conv & $0,04 \pm 0,01^{*}$ & $0,04 \pm 0,01^{*}$ & $0,05 \pm 0,01^{*}$ & $0,04 \pm 0,01^{*}$ & $0,04 \pm 0,01^{*}$ & $0,04 \pm 0,01^{*}$ & $0,06 \pm 0,01^{*}$ \\
\hline Comp & $0,06 \pm 0,01$ & $0,05 \pm 0,01$ & $0,06 \pm 0,01$ & $0,06 \pm 0,01$ & $0,06 \pm 0,01$ & $0,06 \pm 0,01$ & $0,09 \pm 0,10$ \\
\hline Conv & $0,36 \pm 0,05$ & $0,35 \pm 0,06$ & $0,36 \pm 0,05$ & $0,37 \pm 0,04$ & $0,37 \pm 0,05$ & $0,36 \pm 0,05$ & $0,39 \pm 0,04^{*}$ \\
\hline Comp & $0,37 \pm 0,05$ & $0,37 \pm 0,05$ & $0,37 \pm 0,05$ & $0,37 \pm 0,05$ & $0,37 \pm 0,05$ & $0,37 \pm 0,06$ & $0,67 \pm 0,29$ \\
\hline Conv & $0,09 \pm 0,01^{*}$ & $0,09 \pm 0,07$ & $0,08 \pm 0,02$ & $0,10 \pm 0,02$ & $0,08 \pm 0,03$ & $0,08 \pm 0,02$ & $0,09 \pm 0,01^{*}$ \\
\hline Comp & $0,11 \pm 0,01$ & $0,12 \pm 0,06$ & $0,09 \pm 0,02$ & $0,11 \pm 0,02$ & $0,10 \pm 0,02$ & $0,09 \pm 0,02$ & $0,12 \pm 0,02$ \\
\hline
\end{tabular}

Conv: convencional; Comp: computadorizada; BA: base-ápice; * diferença entre os métodos de acordo com o teste de Student (P<0,05).

Horse, assemelhando-se ao descrito para outras espécies, como cães e gatos (WOLF et al., 2000; CAMACHO et al., 2010; GAVA et al., 2011).

Observou-se correlação negativa entre a frequência e a duração dos intervalos PR (computadorizado: $\rho=-0,677$ / $\mathrm{P}<0,001$; convencional: $\quad \rho=-0,405 \quad / \quad \mathrm{P}=0,003), \quad \mathrm{QT}$ (computadorizado: $\quad \rho=-\quad 0,798 \quad / \quad \mathrm{P}<0,001$; convencional: $\rho=-0,765 / \mathrm{P}<0,001)$ em ambos, e correlação positiva entre a frequência e $o$ complexo QRS, somente no método convencional (convencional: $\rho=0,308 / \mathrm{P}=0,002$ ). As correlações negativas entre a frequência cardíaca e os intervalos PR e QT devem-se ao encurtamento do tempo de condução elétrica, em frequências elevadas, através dos átrios e do nodo átrio ventricular (intervalo PR) e pelo feixe de His (intervalo QT).

Neste estudo, não se verificou correlação entre o peso corporal e a frequência cardíaca em nenhum dos métodos (computadorizado: $\rho=-0,267$ / $\mathrm{P}=0,06$; convencional: $\rho=-0,130 / \mathrm{P}=0,366)$, contrariamente ao descrito por SCHWARZWALD et al. (2012). É

Tabela 2 - Amplitude das ondas eletrocardiográficas de equinos da raça American Miniature Horse, submetidos ao eletrocardiograma convencional e computadorizado, segundo as derivações do plano frontal e base-ápice (média \pm desvio-padrão).

\begin{tabular}{|c|c|c|c|c|c|c|c|}
\hline \multicolumn{2}{|c|}{--------- Ondas (mV) I ---------- } & \multirow{2}{*}{$\frac{\text { II }}{0,17 \pm 0,04}$} & \multirow{2}{*}{$\frac{\text { III }}{0,12 \pm 0,04}$} & \multirow{2}{*}{$\frac{\mathrm{aVR}}{-0,12 \pm 0,04}$} & \multirow{2}{*}{$\frac{\mathrm{aVL}}{-0,005 \pm 0,10}$} & \multirow{2}{*}{$\frac{\mathrm{aVF}}{0,13 \pm 0,04}$} & \multirow{2}{*}{$\frac{\mathrm{BA}}{0,21 \pm 0,05}$} \\
\hline P Conv & $0,10 \pm 0,03$ & & & & & & \\
\hline Comp & $0,10 \pm 0,03$ & $0,15 \pm 0,03$ & $0,11 \pm 0,03$ & $-0,12 \pm 0,03$ & $0,004 \pm 0,08$ & $0,13 \pm 0,03$ & $0,22 \pm 0,05$ \\
\hline Conv & $0,09 \pm 0,13$ & $0,20 \pm 0,19$ & $0,30 \pm 0,20$ & $0,19 \pm 0,15$ & $0,12 \pm 0,15$ & $0,22 \pm 0,16$ & $0,02 \pm 0,05$ \\
\hline Comp & $0,06 \pm 0,09$ & $0,21 \pm 0,16$ & $0,30 \pm 0,18$ & $0,19 \pm 0,14$ & $0,08 \pm 0,08$ & $0,23 \pm 0,15$ & $0,02 \pm 0,05$ \\
\hline Conv & - & - & - & - & - & - & $-0,30 \pm 0,30^{*}$ \\
\hline Comp & - & - & - & - & - & - & $-0,66 \pm 0,34$ \\
\hline Conv & $-0,48 \pm 0,29$ & $-0,37 \pm 0,26$ & $0,18 \pm 0,18$ & $0,43 \pm 0,19$ & $-0,27 \pm 0,22$ & $-0,12 \pm 0,22$ & $-0,44 \pm 0,36$ \\
\hline Comp & $-0,51 \pm 0,23$ & $-0,32 \pm 0,31$ & $0,16 \pm 0,26$ & $0,43 \pm 0,21$ & $-0,34 \pm 0,21$ & $-0,07 \pm 0,26$ & $-0,38 \pm 0,44$ \\
\hline
\end{tabular}

Conv: convencional; Comp: computadorizada; BA: base-ápice; * diferença entre os métodos de acordo com o teste de Student $(\mathrm{P}<0,05)$. 
provável que tal relação exista, contudo, o número de animais avaliados talvez tenha sido insuficiente para detectá-la. Como este estudo comparativo entre os métodos é inédito em pôneis, futuramente, mais pesquisas sobre padrões eletrocardiográficos na raça American Miniature Horse são necessárias.

A polaridade da onda $\mathrm{P}$ foi negativa na derivação aVR em ambos os métodos e em todos os animais avaliados neste estudo. Houve predomínio de ondas P bífidas, com dois componentes PI e PII na derivação base-ápice no ECG computadorizado e convencional,sendoestaa configuraçãomaisobservada no traçado eletrocardiográfico do equino também em raças de grande porte. MELCHERT et al. (2012) concluíram que o traçado do ECG computadorizado de cavalos atletas Puro Sangue Lusitano revelou distintas morfologias de ondas $\mathrm{P}$ e complexos QRS, bem como baixa ocorrência de arritmias. A polaridade da onda $\mathrm{T}$ variou entre os métodos, contudo sem diferenças significativas. A porcentagem de ondas $\mathrm{T}$ negativas e bifásicas foi superior no método computadorizado, semelhante ao encontrado por WOLF et al. (2000) e PELTER et al. (1997), que atribuem este fato à acurácia do registro computadorizado, capaz de medir desníveis a partir de $0,01 \mathrm{mV}$.

$\mathrm{O}$ eixo elétrico do complexo QRS não apresentou diferença entre os métodos, sendo a média e desvio-padrão no ECG convencional e computadorizado, respectivamente, de 67,2 $\pm 105,6$ graus e $61,4 \pm 106,6$ graus.

As médias analisadas (duração das ondas e intervalos), obtidas pelos dois métodos eletrocardiográficos foram inferiores às descritas por BUSS et al. (1975) na derivação II, embora a amplitude da onda P (milivolts) tenha se apresentado superior (Tabela 2). Os animais utilizados neste estudo possuíam, entretanto, menor peso corporal (média e desvio-padrão $122,9 \pm 31 \mathrm{~kg}$ ) em relação ao descrito pelos autores (média e desvio-padrão $199 \pm 4 \mathrm{~kg}$ ), fato este que justificaria a diferença entre os valores encontrados.

A literatura é bem ampla quanto à avaliação eletrocardiográfica em equinos de raças de grande porte (FERNANDES et al., 2004; DINIZ et al. 2008; LISBOA et al., 2009; DUMONT et al., 2010; DINIZ et al., 2011), contudo, grande parte dos aspectos eletrocardiográficos descritos ainda não foi estabelecido para os pôneis, particularmente da raça American Miniature Horse. Assim como descrito para outras espécies, há diferenças entre os métodos eletrocardiográficos, convencional e computadorizado, oque exige, portanto, apadronização dos parâmetros em ambos os métodos tanto para a espécie quanto para as raças de baixa estatura.

\section{CONCLUSÃO}

Em conclusão, o presente estudo demonstrou que, em cavalos da raça American Miniature, o exame eletrocardiográfico computadorizado superestimou a duração do complexo QRS em todas as derivações do plano frontal e base-ápice e da amplitude da onda $\mathrm{S}$ nesta última. A presença de correlação negativa entre a frequência cardíaca e os intervalos PR e QT ocorreu em ambos os métodos, e correlação positiva entre frequência e complexo QRS, somente no método convencional.

\section{AGRADECIMENTOS}

Aos proprietários da Fazenda São Benedito/Avaré-SP José Bastos Cruz Sobrinho; Cabanha Guguiná/Lençóis Paulista-SP - Luís Santana Zillo; Haras Jequitibá/Cerqueira César - SP - Marcelo Assumpção Serra; Haras Água Azul/Dois Córregos-SP - José Eden Matosinho.

\section{COMITÊ DE ÉTICA E BIOSSEGURANÇA}

O presente estudo foi aprovado pela Comissão de Ética no Uso de Animais (CEUA), sendo protocolado sob o $n^{\circ} 094 / 2012-$ CEUA.

\section{REFERÊNCIAS}

ASSOCIAÇÃO BRASILEIRA DOS CRIADORES DE MINI-HORSE. Padrão racial do Mini Horse. São Paulo. Online. Disponível em: $<$ http://www.ponei.com.br/arquivos_PDF/Historico_Ficha_Tecnica. pdf $>$. Acesso em: 14. Jan. 2014

BUSS, D.D. et al. The normal electrocardiogram of the domestic pony. Journal of Electrocardiology, v.8, n.2, p.167172, 1975. Disponível em: <http://dx.doi.org/10.1016/S00220736(75)80025-2>. Acesso em: 14 ago. 2014. doi: 10.1016/ S0022-0736(75)80025-2.

CAMACHO, A.A. et al. Comparation between conventional and computadorized electrocardiography in cats. Arquivos Brasileiros de Medicina Veterinária e Zootecnia, v.62, n.3, p.765-769, 2010. Disponível em: <http://dx.doi.org/10.1590/ S0102-09352010000300038>. Acesso em: 14 ago. 2014. doi:10.1590/S0102-09352010000300038.

DINIZ, M.P. et al. Estudo eletrocardiográfico de equinos da raça Mangalarga Marchador. Arquivos Brasileiros de Medicina Veterinária eZootecnia, v.60, n.3,p.536-542,2008. Disponível em: $<$ http://dx.doi.org/10.1590/S0102-09352008000300003>. Acesso em: 14 ago. 2014. doi: 10.1590/S0102-09352008000300003.

DINIZ, M.P. et al. Estudo eletrocardiográfico de equinos de salto sadios. Pesquisa Veterinária Brasileira, v.31 n.4, p. 355361, 2011. Disponível em: <http://dx.doi.org/10.1590/S0100736X2011000400014>. Acesso em: 14 ago. 2014. doi: 10.1590/ S0100-736X2011000400014.

DUMONT, C.B.S. et al. Parâmetros eletrocardiográficos de equinos Puro Sangue Árabe submetidos a exercício prolongado de enduro. Ciência Rural, v.40, n.9, p.19661973, 2010. Disponível em: <http://dx.doi.org/10.1590/ 
S0103-84782010000900018>. Acesso em: 14 ago. 2014. doi: $10.1590 / \mathrm{S} 0102-09352004000200002$.

FERNANDES, W.R. et al. Características eletrocardiográficas em equinos clinicamente normais da raça Puro Sangue Inglês. Arquivos Brasileiros de Medicina Veterinária e Zootecnia, v.56, n.2, p.143-149, 2004. Disponível em: <http://dx.doi.org/10.1590/ S0102-09352004000200002>. Acesso em: 14 ago. 2014. doi: 10.1590/S0102-09352004000200002.

FREGIN, G.F. The equine electrocardiogram with standardized body and limb positions. Cornell Veterinarian, v.72, p.304-324, 1982.

GAVA, F.N. et al. Eletrocardiografia computadorizada em cães da raça Beagle. Arquivos Brasileiros de Medicina Veterinária e Zootecnia, v.63, n.2, p.317-321, 2011. Disponível em: <http:// dx.doi.org/10.1590/S0102-09352011000200007>. Acesso em: 14 ago. 2014. doi: 10.1590/S0102-09352011000200007.

HILWIG, R. Cardiac arrhythmias in the horse. Journal of American Veterinary Medical Association, v.170, p.153-163, 1977.

HONGO, R.H.; GOLDSCHLAGER, N. Status of computerized electrocardiography. Cardiology Clinics, v.24, p.491-504, 2006. Disponível em: <http://dx.doi.org/10.1016/j.ccl.2006.03.005>. Acesso em: 14 ago. 2014. doi: 10.1016/j.ccl.2006.03.005.

LESSA, D.F.S. et al. Considerações sobre o uso da Eletrocardiografia e sua Interpretação em Equinos. Revista Brasileira de Medicina Equina, v.42, p.4-12, 2012.

LISBOA, F.P. et al. Dados preliminares do padrão eletrocardiográfico de equinos da raça Crioula. In: CONGRESSO DE INICIAÇÃO CIENTÍfICA, 28., 2009, Pelotas, RS. Anais... Pelotas: CICX VIII, 2009. Disponível em: $<$ http://www.ufpel.edu. br/cic/2009/cd/pdf/CA/CA_02098.pdf>. Acesso em: 14 jan. 2014.

MELCHERT, A. et al. Eletrocardiografia computadorizada em cavalos Puro Sangue Lusitano submetidos a exercício físico. Arquivo Brasileiro de Medicina Veterinária, v.64, n.3, p.547-554, 2012. Disponível em: <http://www.scielo.br/ pdf/abmvz/v64n3/104.pdf>. Acesso em: 14 abr. 2014. doi: 10.1590/S0102-09352012000300004.

PELTER, M.M. et al. Computer versus manual measurement of ST-segment deviation. Journal of Electrocardiology, v.30, p.151-156, 1997. Disponível em: <http://dx.doi.org/10.1016/ S0022-0736(97)80025-8>. Acesso em: 14 abr. 2014. doi: 10.1016/ S0022-0736(97)80025-8.

LOON, G.; PATTESON, M.W. Electrophysiology and arrhythmogenesis. In: MARR, C.M.; BOWEN, I.M. Cardiology of the horse. Toronto: Saunders Elsevier, 2010. Cap.6, p.59-73.

REEF, V.B. Cardiovascular problems associated with poor performance. In: ROBINSON, N.E. (Ed). Current therapy in equine medicine. London: Saunders, 1992. p.381-410.

REZAKHANI, A. et al. Effects on the prevalence of cardiac dysrhythmias in ponies. Iranian Journal of Veterinary Research, v.11, n.3, p.200-205, 2010. Disponível em: <http:// ijvr.shirazu.ac.ir/pdf_120_dbac1189980336fd62f06065f2e18ea3. html >. Acesso em: $1 \overline{4}$ ago. 2014.

ROBERTSON, S.A. Electrocardiography for the equine practioner. Veterinary Annual, v.32, p.192-200, 1992. Disponível em:<file://F:/ Documentos\%201/Projetos\%20de\%20Pesquisa/TELEVET\%20100/ art79501.pdf $>$. Acesso em: 14 ago. 2014.

SCHWARZWALD, C.C. et al. Relationship of heart rate and electrocardiographic time intervals to body mass in horses and ponies. Journal of Veterinary Cardiology, v.14, p.343-350, 2012. Disponível em: <http://www.sciencedirect.com/science/ article/pii/S1760273412000537\#dcbf $>$. Acesso em: 14 abr. 2014. doi: 10.1016/j.jvc.2012.03.001.

SHIWEN, Y. et al. Clinical application of a microcomputer system for analysis of monophasic action potentials. Pacing and Clinical Electrophysiology, v.19, p.297-308, 1996. Disponível em: <http:// onlinelibrary.wiley.com/doi/10.1111/j.1540-8159.1996.tb03331.x/pdf>. Acesso em: 14 abr. 2014. doi: 10.1111/j.1540-8159.1996.tb03331.x.

TILLEY, L.P. Essentials of canine and feline electrocardiography: interpretation and treatment. Philadelphia: Lea \& Febiger, 1992. 470p.

WOLF, R. et al. Eletrocardiografia computadorizada em cães. Arquivos Brasileiros de Medicina Veterinária e Zootecnia, v.52, p.610-615, 2000. Disponível em: < http://www. scielo.br/scielo.php?pid=S0102-09352000000600010\&script $=$ sci arttext $>$. Acesso em: 14 abr. 2014. doi: 10.1590/S010209352000000600010 . 